\title{
An Educational Intervention to Improve the Sleep Behavior and Well-Being of High School Students
}

Alexandra Colt, BA I Jo Marie Reilly, MD, MPH

PRIMER. 2019;3:21.

Published: 9/26/2019 | DOI: 10.22454/PRiMER.2019.871017

\section{Abstract}

Objective: The objective of this study was to determine whether a sleep education intervention improves knowledge of sleep, sleep behaviors, and depression in high school freshmen.

Methods: We recruited student volunteers at a single magnet high school in Los Angeles, California through their health class. Twenty-four freshmen participated and 18 students (17 female, 1 male) completed pre- and postsurveys. Curriculum consisted of 4 hours of after-school interactive lectures emphasizing sleep physiology, benefits of sleep, what impacts sleep, and methods to improve sleep, followed by a 9-week sleep behavior change journal. Pre- and postsurveys measuring both sleep behaviors and knowledge, and a Patient Health Questionnaire-9 depression screening were administered to participants prior to and after the intervention. We used $t$ tests and $\chi^{2}$ tests to analyze knowledge and behavior change.

Results: Subjects improved in average sleep hours per night (preintervention 6.9 hours to postintervention 7.8 hours, $P=.0134)$, and average weekend night bedtime (11:36 pm to $10: 54 \mathrm{pm}, P=.0307)$.

Conclusions: This school sleep behavior intervention demonstrated students' average sleep hours per night and weekend bedtime improved after the lecture and sleep journal intervention. This suggests a sleep education intervention may benefit this population. Further studies are needed to demonstrate effectiveness of this education over time, across sexes, and in high-risk students.

\section{Introduction}

Teenage students, especially those in competitive academics, are at risk for sleep deprivation. ${ }^{1}$ This can harm health, mood, and academics. ${ }^{2}$ The current recommendation from the National Sleep Foundation is 8 to 10 hours of sleep per night for adolescents. ${ }^{3}$ Further, adolescents' melatonin-the "sleep hormone"-often does not release until late at night and peaks in the early morning. ${ }^{4}$ This causes them to be more awake later in the day and more tired when they need to get up for school. The shift in circadian rhythm makes it difficult for teens to adapt their sleep schedule to a normal school day and to get the recommended 8-10 hours of sleep per night. The biological change in sleep, coupled with academic pressure to get homework done, often influences students to stay up later. The net effect is that students are sleepy at school. ${ }^{1}$ Weekend catch-up sleep is problematic, too, as it forces adolescents out of their circadian rhythm. ${ }^{5}$

In addition to considering the biological and schedule factors that impact adolescent sleep, it is also important to consider the impacts of bedtime autonomy, caffeine, physical activity, friends, homework, electronics, noise, and responsibilities at home. Researchers in the field of adolescent sleep have highlighted the importance of sleep education and encouragement to make healthy sleep choices, ${ }^{6,7}$ especially regarding electronics usage that cause sleep difficulties. Additionally, electronics usage has been linked to depressive symptoms. ${ }^{8}$ 
The sleep deficit in this population is significant enough that the United States government initiative, Healthy People 2020, includes "increase the proportion of students in grades 9 through 12 who get sufficient sleep" as one of its four sleep health objectives. Healthy People 2020 reports that in 2009 , only $30.9 \%$ of students in these grades got sufficient sleep. ${ }^{9}$ As screen time has suddenly increased and the duration of other distracting behaviors has remained relatively stable, the percentage of adolescents sleeping 7 hours per night or less has also increased, suggesting an impact of electronics usage on sleep behaviors. ${ }^{10,11}$

Although this population often has knowledge of the importance of sleep, they are still sleep deprived, due to lack of knowledge of sleep-improving tactics and corresponding sleep-preserving behaviors. Sleep also determines whether electronics usage affects depression in this age group as electronics usage only correlates with depression when subjects are also low on sleep or have problems sleeping ${ }^{12}$; and sleep problems from adolescence have been found to persist into adulthood, ${ }^{13}$ highlighting the need for early intervention in sleep education.

Lack of sleep had been identified by students as a problem at Francisco Bravo Medical Magnet High School by afterschool program leaders. In response, a course was created to educate students about sleep behavior and physiology. We hypothesized that after an educational sleep intervention, subjects would report increased sleep time per night, decreased depression, ${ }^{14}$ and improved knowledge regarding sleep behavior and physiology.

\section{Methods}

The institutional review board at the University of Southern California approved this study (HS-16-00715), and parents/guardians provided consent using a standard consent form in English and Spanish.

\section{Subjects}

The assistant principal at Francisco Bravo Medical Magnet School in Los Angeles, California recruited high school freshmen through their freshman health class. Student participants were given community service hour credit for each hour of participation; these hours are required for graduation. Freshmen were chosen so the intervention could have the longest possible impact on their high school careers. Twenty-four subjects participated in the intervention, and 18 completed the presurvey, lecture, journal, and postsurvey and were included in the data analysis. The average age of participants was 14 years.

\section{Sleep Intervention}

The sleep intervention consisted of one 2-hour and two 1-hour after-school courses created and taught by the principal investigator. The curriculum was designed to teach subjects about sleep physiology and its importance, its impact on health, and methods to improve sleep hygiene and sleep behaviors. The teaching consisted of interactive lectures with PowerPoint slides. The first 2-hour class included information about basic sleep physiology (ie, functions of melatonin, caffeine, neurotransmitters) and sleep recommendations by age. ${ }^{15}$ At the end of the first class, subjects were asked to write down what they would like to learn in the course, and their answers were the focus of the second class.

Topics for the second module included strategies for time management, sleeping through the night, sleeping when not tired, and sleeping more hours. ${ }^{16}$ Between the second and third class, the subjects were given sleep behavior change journals to complete over 9 weeks. The sleep behavior change journal asked subjects to record how many hours they slept each night. It also asked them to set a personal behavior change goal and write each week about how they adhered to their goal, what made it difficult, what they could do to improve, and what their mood was like that week. Suggestions of goals for subjects to choose included (1) keep a sleep schedule (same time to bed and to awaken), (2) do something relaxing (nonelectronic, eg, reading or meditation) before bed, (3) turn off electronics 1 hour before bed, (4) exercise regularly, (5) avoid caffeine, (6) make room darker and temperature cooler, and (7) keep the bed just for sleep. ${ }^{17}$ Subjects were also asked to compare their sleep behavior to that of the previous weeks and note any changes they had made. The course and journal times and durations were selected to best match the school's and subjects' schedules. 


\section{Survey Instrument}

We used a pre- and postintervention sleep survey to record students' sleep behavior. ${ }^{18}$ The survey asked about sleep hours per night, school night and weekend night bed times, reasons for not sleeping more, hours spent on homework, sleep and wake aids, whether there was competition regarding sleep among friends and at school, whether subjects and their families thought they slept enough, use of technology prior to bedtime, and whether they were worried about their sleep. These topics were included to gather basic sleep statistics about the cohort and to assess students' basic knowledge about sleep hygiene pre- and postintervention.

The pre- and postsurveys also evaluated students' knowledge about adolescent sleep behavior and physiology. These questions asked about (1) how many hours teenagers should sleep, (2) how sleep affects academic performance, risk-taking, obesity, mood, and caffeine intake, (3) electronics usage, (4) food consumption and physical activity, and (5) depression and anxiety and their impact on sleep. The preintervention survey also asked what subjects would like to see included in the course. The postintervention survey asked if subjects believed the course would affect their long-term behavior, what did and did not work about the course, what they would like to see changed in future iterations of the course, and the top three things they learned from the course. The survey instrument included a depression screening (Patient Health Questionnaire-9 [PHQ-9], a validated depression screening instrument).

\section{Statistical Analysis}

The principal investigator collected and analyzed all data and was blinded to subject survey responses. Of the 24 subjects recruited, 18 (17 female, 1 male) completed the preintervention survey, lecture, journal, and postintervention survey and were included in the data analysis. The remining six students were unable to complete all parts of the course due to scheduling conflicts. We used $t$ tests and $\chi^{2}$ tests to analyze pre- to posttest change.

\section{Results}

\section{Sleep Behavior Change}

Subjects reported an increase in average sleep hours per school night (preintervention 6.9 hours to postintervention 7.8 hours, $P=.0134$; Figure 1, with $50 \%$ of students reporting an increase in average hours slept per night) and in average weekend night bedtime $(11: 36 \mathrm{pm}$ to $10: 54 \mathrm{pm}, P=.0307$; Figure 2 , with $44 \%$ of students reporting an earlier average bedtime).

There was no statistically significant pre- to postintervention change in night-before-survey sleep hours, school night bedtime, weekend sleep hours, school night homework hours, or sleep hours desired.

In a postintervention discussion led by the principal investigator, the cohort also reported increased use of a consistent bedtime, both on school and weekend nights, a key teaching point. There was an increase-although not statistically significant-in the proportion of subjects who thought they slept enough before the course: $33 \%$ (6 subjects), compared to the proportion of subjects $61 \%$ (11) who thought so after course completion. At the end of the course, $89 \%$ (16) thought the course would affect their long-term sleep behavior.

\section{Sleep Knowledge}

The understanding of the number of hours teenagers should sleep (8-10 hours per night) decreased, as the number of students who corretly answered the multiple-choice question about the number of hours teenagers should sleep (8-10 per night) decreased (13 to $7, P=.0442)$.

There was no statistically significant change pre- to postintervention in the percentage of correct multiple-choice answers to the following survey questions ${ }^{18}$ : (1) How does sleep affect academic performance? (2) How does sleep time affect risk-taking behavior? (3) How does sleep time affect obesity? (4) How does caffeine affect sleep? (5) How does using technology affect sleep? (6) How does food affect sleep? (7) How does physical activity affect sleep? (8) How does depressed or anxious mood affect sleep? (9) How does sleep affect mood? 


\section{Well-Being}

Those who scored $\geq 10$ (moderate or more severe depression) on the PHQ-9, or indicated suicidal ideation, were reidentified and referred to the school psychologist for immediate mental health intervention (six subjects preintervention, two subjects postintervention). PHQ-9 scores decreased over the course of the intervention, but not in a statistically significant way. The change in percentage of subjects with moderate or more severe depression preintervention to postintervention decreased, but was not statistically significant either.

\section{Discussion}

Adolescent sleep hygiene is an area of importance to health care providers, as it impacts adolescent health, wellbeing, and academic performance. Developing tools that can improve sleep hygiene is important to improving the overall well-being of this population. This pilot study used a sleep behavior and physiology course and a sleep journal in an effort to improve adolescent sleep knowledge, behavior, and overall well-being. Enrolled subjects were interested in learning about and improving their sleep.

The study resulted in statistically significant improvements in average sleep hours and average weekend night bedtimes, suggesting adolescent understanding of the need for more sleep and the need for consistent bed times every night, including weekends. However, there was a decrease in understanding of the number of hours teenagers should sleep (8-10 hours per night). This may indicate the need to further emphasize this point. Subjects identified the sleep behavior change journals as an important factor in their ability to improve their sleep habits, suggesting that sleep knowledge is most likely not enough to achieve behavior change, rather, an interactive task was instrumental in habit improvement.

Many subjects identified electronics as a reason for not sleeping more, and use of electronics was the most common area in which students hoped to change behavior in the future. Although electronics are an important part of an adolescent's life, teens are motivated to change their relationship with electronics in order to improve their sleep. In fact, the most common goal for change after this course was no electronics usage before bed (six subjects). Increased screening tools, education, and intervention regarding the use of electronics and their impact on sleep may be necessary.

Most participant reviews of the course were favorable. Subjects identified the most favorable aspects of the course as sleep behavior information/recommendations (11 subjects), learning sleep physiology/the effects of sleep (5), and the sleep behavior change journal helping subjects stick to their goals (4 subjects). The teens identified the most difficult things about the course as: difficulty sticking to the goals subjects set for themselves during the behavior change project (3), only having three course sessions (3), and the length of the 9-week sleep behavior change project and journal (1). When asked the top three things students learned from the course, subjects reported: caffeine physiology (10), sleep's effect on academics (8), electronics' effect on sleep (7), melatonin/internal clock function (6), and teen sleep physiology (4). These reviews will help inform and improve future iterations of teen sleep studies.

Limitations of this study include sample size (18), subject gender (17 female, 1 male), and self-report survey method. It would be ideal to have a larger sample size and retain a larger portion of subjects, as the six subjects who were not included in the statistical analysis were unable to finish one or more of the parts of the course. A longitudinal follow-up survey would inform whether the lessons and effects of this course persist.

Other limitations of this study include intrinsic and extrinsic bias in the pre- and postsurveys, sleep journal reporting, and data collection. Specifically, on the latter, the principal investigator handled all elements of the course and data analysis. In future studies, another investigator should assess subjects in order to avoid bias in subject responses. Additionally, those students who did not complete all parts of the study may have had a lower survey response. Eliminating them from the data analysis may have skewed the study results. 
Future studies should include (1) a larger sample size with an even sex ratio, (2) varying the amount of teaching hours to see whether subjects' degree of improvement in sleep behaviors is dose-dependent, and (3) investigation into the effects on sleep of bedtime autonomy, physical activity, noise, and responsibilities at home. A follow-up study could also include a sleep-tracking application (to minimize the subjectivity of self-report mechanisms) and monitoring of smartphone usage hours (to examine usage effect on sleep).

Adolescents appear to be receptive to sleep education and sleep behavior change. These changes may also improve their sleep knowledge and well-being. Those in the adolescent medicine, sleep medicine, and school health fields should consider the importance of sleep behavior and physiology education in their counseling of adolescents.

\section{Tables and Figures}

Figure 1: Average Hours Slept on a School Night Pre- to Postintervention

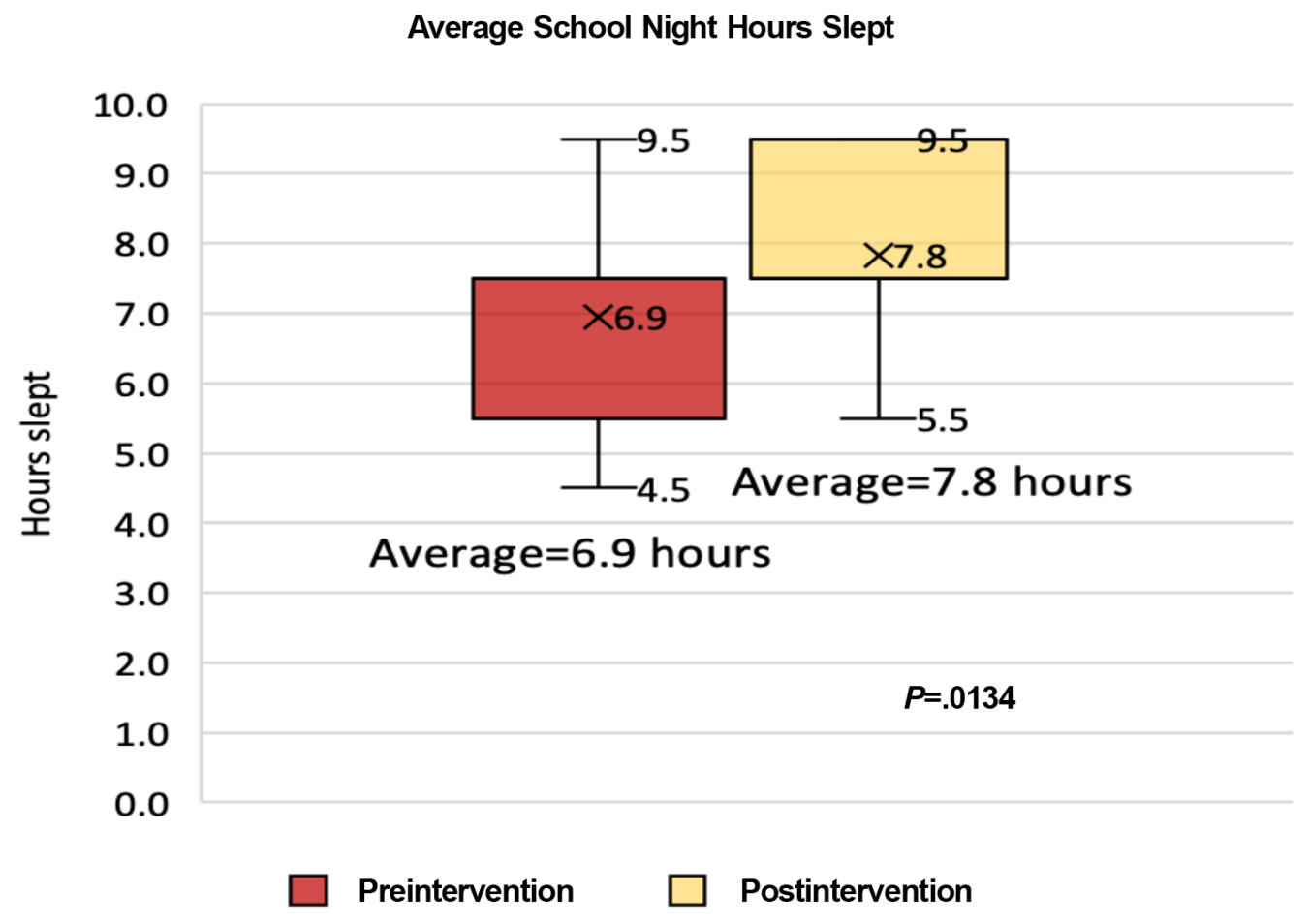


Figure 2: Average Time Subjects Went to Sleep Preintervention to Postintervention

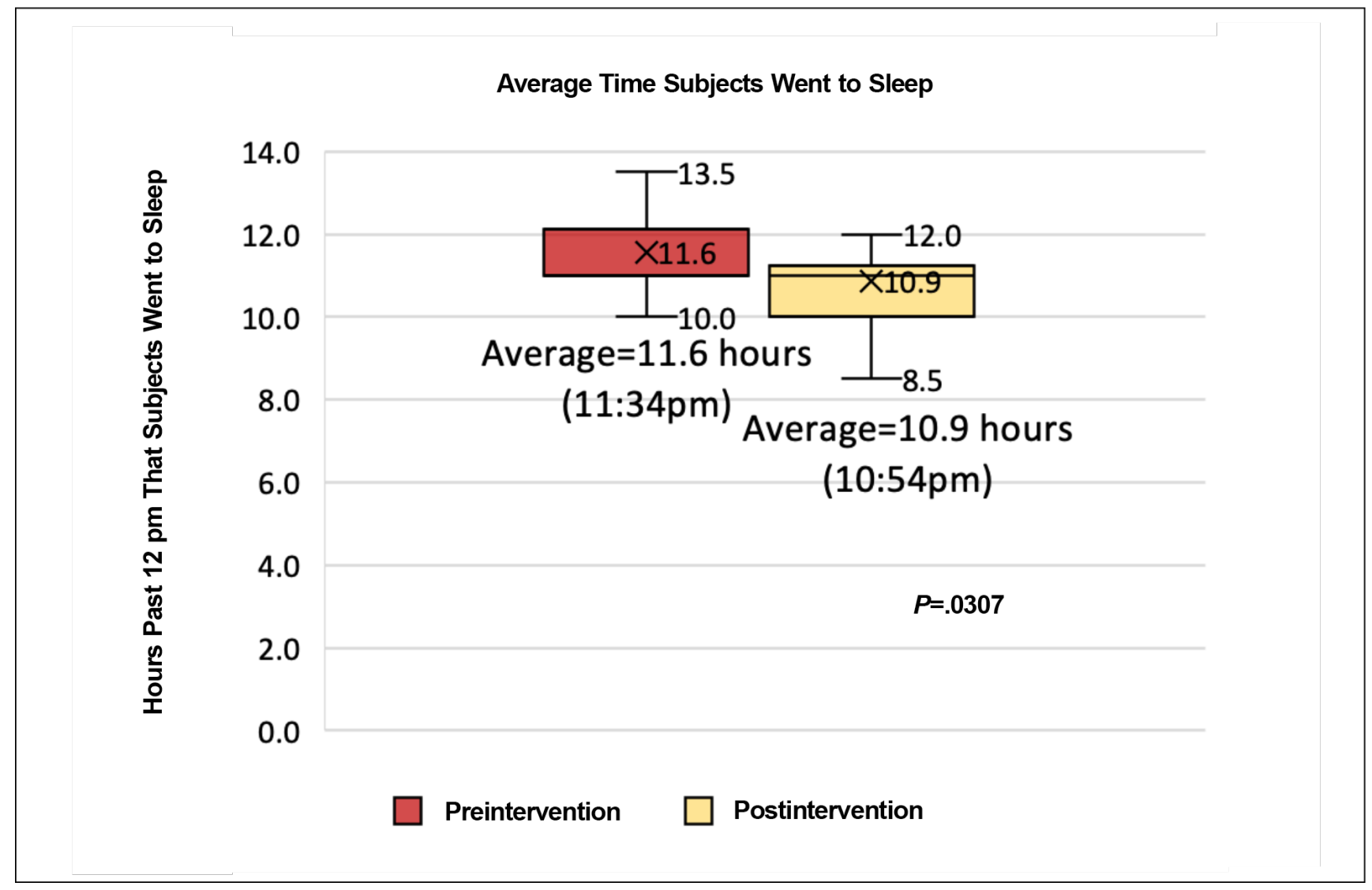

\section{Corresponding Author}

Alexandra Colt, BA

Keck School of Medicine of the University of Southern California, 1975 Zonal Avenue, KAM B29, Los Angeles, CA 90033.

acolt@usc.edu

\section{Author Affiliations}

Alexandra Colt, BA - Keck School of Medicine of the University of Southern California, Los Angeles, CA Jo Marie Reilly, MD, MPH - Keck School of Medicine of the University of Southern California, Los Angeles, CA

\section{References}

1. Carskadon MA. Patterns of sleep and sleepiness in adolescents. Pediatrician. 1990;17(1):5-12.

2. Carskadon MA, Acebo C, Jenni OG. Regulation of adolescent sleep: implications for behavior. Ann N Y Acad Sci. 2004 Jun;1021:276-91. https://doi.org/10.1196/annals.1308.032

3. Hirshkowitz M, Whiton K, Albert SM, et al. National Sleep Foundation's updated sleep duration recommendations: final report. Sleep Health. 2015;1(4):233-243. https://doi.org/10.1016/j.sleh.2015.10.004

4. Carskadon MA, Acebo C, Richardson GS, Tate BA, Seifer R. An approach to studying circadian rhythms of adolescent humans. J Biol Rhythms. 1997;12(3):278-289. https://doi.org/10.1177/074873049701200309

5. Crowley SJ, Acebo C, Carskadon MA. Sleep, circadian rhythms, and delayed phase in adolescence. Sleep Med. 2007 Sep;8(6):602-12. https://doi.org/10.1016/j.sleep.2006.12.002

6. Carskadon MA. Sleep in adolescents: the perfect storm. Pediatr Clin North Am. 2011. https://doi.org/10.1016 /j.pcl.2011.03.003.

7. Owens J; Adolescent Sleep Working Group; Committee on Adolescence. Insufficient sleep in adolescents and young adults: an update on causes and consequences. Pediatrics. 2014;134(3):e921-e932. https://doi.org 
/10.1542/peds.2014-1696

8. Lemola S, Perkinson-Gloor N, Brand S, Dewald-Kaufmann JF, Grob A. Adolescents' electronic media use at night, sleep disturbance, and depressive symptoms in the smartphone age. J Youth Adolesc.

2015;44(2):405-418. https://doi.org/10.1007/s10964-014-0176-x

9. Office of Disease Prevention and Health Promotion. Sleep Health, Healthy People 2020 Topics and Objectives. www.healthypeople.gov/2020/topicsobjectives2020/overview.aspx?topicid=38. Accessed April 1, 2019.

10. Twenge JM, Krizan Z, Hisler G. Decreases in self-reported sleep duration among US adolescents 2009-2015 and association with new media screen time. Sleep Med. 2017;39:47-53. https://doi.org/10.1016 /j.sleep.2017.08.013

11. Hysing M, Pallesen S, Stormark KM, Jakobsen R, Lundervold AJ, Sivertsen B. Sleep and use of electronic devices in adolescence: results from a large population-based study. BMJ Open. 2015;5(1):e006748. https://doi.org/10.1136/bmjopen-2014-006748

12. Li X, Buxton OM, Lee S, Chang AM, Berger LM, Hale L. Sleep mediates the association between adolescent screen time and depressive symptoms. Sleep Med. 2019;57:51-60. https://doi.org/10.1016 /j.sleep.2019.01.029

13. Fatima Y, Doi SAR, Najman JM, Al Mamun A. Continuity of sleep problems from adolescence to young adulthood: results from a longitudinal study. Sleep Health. 2017;3(4):290-295. https://doi.org/10.1016 /j.sleh.2017.04.004

14. Berger AT, Wahlstrom KL, Widome R. Relationships between sleep duration and adolescent depression: a conceptual replication. Sleep Health. 2019;5(2):175-179. https://doi.org/10.1016/j.sleh.2018.12.003

15. National Institute of Neurological Disorders and Stroke. Brain Basics: Understanding Sleep. www.ninds.nih.gov/Disorders/Patient-Caregiver-Education/Understanding-Sleep. Updated February 8, 2019. Accessed September 16, 2019.

16. Garey, Juliann. How to Help Teenagers Get More Sleep. Child Mind Institute Topics A-Z. https://childmind.org /article/help-teenagers-get-sleep/. Accessed April 1, 2019.

17. Division of Sleep Medicine at Harvard Medical School. Adopt Good Sleep Habits. Get Sleep; 2008. https://www.healthysleep.med.harvard.edu/need-sleep/what-can-you-do/good-sleep-habits. Accessed September 16, 2019.

18. Colt A, Reilly JM. Sleep Course Pre- and Post-Intervention Surveys. STFM Resource Library; April 12, 2019. https://resourcelibrary.stfm.org/viewdocument/sleep-course-pre-interventionsurve?CommunityKey=2751b51d-483f-45e2-81 de-4faced0a290a\&tab=librarydocuments. Accessed September 16, 2019.

Copyright $\odot 2019$ by the Society of Teachers of Family Medicine 